\title{
A Gronwall inequality via the generalized proportional fractional derivative with applications
}

\author{
Jehad Alzabut ${ }^{* *}$, Thabet Abdeljawad ${ }^{1}$, Fahd Jarad ${ }^{2}$ and Weerawat Sudsutad ${ }^{3}$
}

"Correspondence:

jalzabut@psu.edu.sa

${ }^{1}$ Department of Mathematics and

General Sciences, Prince Sultan

University, Riyadh, Saudi Arabia

Full list of author information is

available at the end of the article

\section{Springer}

\begin{abstract}
In this paper, we provide a new version for the Gronwall inequality in the frame of the generalized proportional fractional derivative. Prior to the main results, we introduce the generalized proportional fractional derivative and expose some of its features. As an application, we accommodate the newly defined derivative to prove the uniqueness and obtain a bound in terms of Mittag-Leffler function for the solutions of a nonlinear delay proportional fractional system. An example is presented to demonstrate the applicability of the theory.
\end{abstract}

Keywords: Proportional Gronwall inequality; Uniqueness of solution; Delay proportional fractional system; Bound for the solution

\section{Introduction}

Integral inequalities are a fabulous instrument for developing the qualitative and quantitative properties of differential equations. There has been a continuous growth of interest in such an area of research in order to meet the needs of various applications of these inequalities. Such inequalities have been studied by many researches who in turn used diverse techniques for the sake of exploring and proposing these inequalities [1-3]. One of the most important inequalities is the distinguished Gronwall inequality [4-8].

On the other hand, the fractional calculus, which is considered to be the generalization of the traditional calculus dealing with nonnegative integer order integration and differentiation, has been one of the most rapidly growing fields of research because of the interesting results ascertained when the fractional operators are applied to model real world problems [9-14]. An attracting particularization of this field is that there are many fractional operators, and this allows the researchers to select the most suitable operator for the sake of modeling the problem under investigation. Besides, because of its simplicity in applications, researchers have paid more attention to recently discovered fractional operators without singular kernels $[15,16]$, and then many articles considering these types of fractional operators have recently come into view.

In parallel to the increasing interests in the theory of fractional differential equations, many researchers have extended these mathematical inequalities to differential equations containing fractional derivatives involving singular and nonsingular kernels. We refer here to some of these results [17-28]. Following this tendency, we provide a new version for

(c) The Author(s) 2019. This article is distributed under the terms of the Creative Commons Attribution 4.0 International License (http://creativecommons.org/licenses/by/4.0/), which permits unrestricted use, distribution, and reproduction in any medium, provided you give appropriate credit to the original author(s) and the source, provide a link to the Creative Commons license, and indicate if changes were made. 
Gronwall inequality in the frame of the generalized proportional fractional (GPF) derivatives. More precisely, we prove the following result: If we have

$$
u(t) \leq v(t)+\rho^{\alpha} \Gamma(\alpha) w(t)\left({ }_{0} I^{\alpha, \rho} u\right)(t)
$$

then

$$
u(t) \leq v(t)+\int_{0}^{t}\left\{\sum_{m=1}^{\infty} \frac{(w(t) \Gamma(\alpha))^{m}}{\Gamma(m \alpha)} e^{\frac{\rho-1}{\rho}(t-s)}(t-s)^{m \alpha-1} v(s)\right\} d s, \quad t \in[0, T),
$$

where ${ }_{0} I^{\alpha, \rho}$ is the proportional fractional integral of order $\alpha, \rho>0, u, v$ are nonnegative locally integrable functions on $[0, T)$, and $w(t)$ is a nonnegative, nondecreasing, and continuous function defined on $t \in[0, T)$ such that $w(t) \leq M$, where $M$ is a constant. The peculiarity of the proportional fractional operators is that their kernels contain an exponential term that comes out naturally in addition to the kernel that appears in the traditional fractional operators.

The structure of the paper is organized as follows: Sect. 2 is devoted to providing essential preliminaries on the GPF derivatives and integrals as well as stating some fundamental lemmas that will be used in the subsequent sections. Section 3 provides the Gronwall inequality within the GPF derivatives. Section 4 discusses some applications on the uniqueness of solutions for a delay system. Moreover, a bound for the solutions is also provided. An example that examines the theoretical results is provided at the end of the paper. Section 5 concludes our results.

\section{Preliminaries on GPF derivative and integral}

In this section, we set forth some terminologies, definitions, and essential lemmas that will be used throughout the remaining part of the paper. For their justifications and proofs, the reader can consult the paper [29].

A limit-based definition of the so-called conformable derivative was given in [30,31] as follows:

$$
D^{\alpha} f(t)=\lim _{\epsilon \rightarrow 0} \frac{f\left(t+\epsilon t^{1-\alpha}\right)-f(t)}{\epsilon} .
$$

It is obvious that when the function $f$ is differentiable, the conformable derivative of $f$ reads

$$
D^{\alpha} f(t)=t^{1-\alpha} f^{\prime}(t)
$$

The main drawback of this derivative is that when the order of the derivative is 0 or $\alpha \rightarrow 0$, the function $f$ is not obtained. That is, $D^{0} f \neq f$. In order to overcome this problem and benefit from the proportional derivative for controller output with two tuning parameters [32], Anderson et al. defined the modified conformable derivative as follows.

Definition 1 For $\rho \in[0,1]$, let the functions $\kappa_{0}, \kappa_{1}:[0,1] \times \mathbb{R} \rightarrow[0, \infty)$ be continuous such that, for all $t \in \mathbb{R}$, we have

$$
\lim _{\rho \rightarrow 0^{+}} \kappa_{1}(\rho, t)=1, \quad \lim _{\rho \rightarrow 0^{+}} \kappa_{0}(\rho, t)=0, \quad \lim _{\rho \rightarrow 1^{-}} \kappa_{1}(\rho, t)=0, \quad \lim _{\rho \rightarrow 1^{-}} \kappa_{0}(\rho, t)=1,
$$


and $\kappa_{1}(\rho, t) \neq 0, \rho \in[0,1), \kappa_{0}(\rho, t) \neq 0, \rho \in(0,1]$. Then, the proportional derivative of order $\rho$ is defined by

$$
D^{\rho} f(t)=\kappa_{1}(\rho, t) f(t)+\kappa_{0}(\rho, t) f^{\prime}(t) .
$$

For more details about the control theory of the proportional derivative and its component functions $\kappa_{0}$ and $\kappa_{1}$, we refer the reader to [32,33]. We shall restrict ourselves to the case when $\kappa_{1}(\rho, t)=1-\rho$ and $\kappa_{0}(\rho, t)=\rho$. Therefore, (5) becomes

$$
D^{\rho} f(t)=(1-\rho) f(t)+\rho f^{\prime}(t) .
$$

It is easy to figure out that $\lim _{\rho \rightarrow 0^{+}} D^{\rho} f(t)=f(t)$ and $\lim _{\rho \rightarrow 1^{-}} D^{\rho} f(t)=f^{\prime}(t)$. Thus, the derivative (6) is somehow considered to be more general than the conformable derivative which evidently does not tend to the original functions as $\rho$ tends to 0 .

The GPF integral and derivative are defined as follows.

Definition 2 ([29]) For $0<\rho \leq 1, \alpha \in \mathbb{C}$, and $\operatorname{Re}(\alpha)>0$, the GPF integral of $f$ of order $\alpha$ is

$$
\left({ }_{a} I^{\alpha, \rho} f\right)(t)=\frac{1}{\rho^{\alpha} \Gamma(\alpha)} \int_{a}^{t} e^{\frac{\rho-1}{\rho}(t-\tau)}(t-\tau)^{\alpha-1} f(\tau) d \tau=\rho^{-\alpha} e^{\frac{\rho-1}{\rho} t}\left({ }_{a} I^{\alpha}\left(e^{\frac{1-\rho}{\rho} t} f(t)\right)\right) .
$$

Definition 3 ([29]) For $0<\rho \leq 1, \alpha \in \mathbb{C}, \operatorname{Re}(\alpha) \geq 0$, and $n=[\operatorname{Re}(\alpha)]+1$, then the GPF derivative of $f$ of order $\alpha$ is

$$
\left({ }_{a} D^{\alpha, \rho} f\right)(t)=\left(D^{n, \rho}{ }_{a} I^{n-\alpha, \rho} f\right)(t)=\frac{D_{t}^{n, \rho}}{\rho^{n-\alpha} \Gamma(n-\alpha)} \int_{a}^{t} e^{\frac{\rho-1}{\rho}(t-\tau)}(t-\tau)^{n-\alpha-1} f(\tau) d \tau .
$$

If we let $\rho=1$ in Definition 3, then one can obtain the left Riemann-Liouville fractional derivative $[11,13,14]$. Moreover, it is obvious that

$$
\lim _{\alpha \rightarrow 0}\left(D^{\alpha, \rho} f\right)(t)=f(t) \quad \text { and } \quad \lim _{\alpha \rightarrow 1}\left(D^{\alpha, \rho} f\right)(t)=\left(D^{\rho} f\right)(t) .
$$

Proposition 1 ([29]) Let $\alpha, \beta \in \mathbb{C}$ be such that $\operatorname{Re}(\alpha) \geq 0$ and $\operatorname{Re}(\beta)>0$. Then, for any $\rho>0$, we have

(1) $\left({ }_{a} I^{\alpha, \rho} e^{\frac{\rho-1}{\rho} t}(t-a)^{\beta-1}\right)(x)=\frac{\Gamma(\beta)}{\Gamma(\beta+\alpha) \rho^{\alpha}} e^{\frac{\rho-1}{\rho} x}(x-a)^{\alpha+\beta-1}, \operatorname{Re}(\alpha)>0$.

(2) $\left({ }_{a} D^{\alpha, \rho} e^{\frac{\rho-1}{\rho} t}(t-a)^{\beta-1}\right)(x)=\frac{\rho^{\alpha} \Gamma(\beta)}{\Gamma(\beta-\alpha)} e^{\frac{\rho-1}{\rho} x}(x-a)^{\beta-1-\alpha}, \operatorname{Re}(\alpha) \geq 0$.

In the following lemmas, we expose some features of the GPF operator.

Lemma 1 ([29]) If $\rho>0, \operatorname{Re}(\alpha)>0$, and $\operatorname{Re}(\beta)>0$, then, for $f$ is continuous and defined for $t \geq a$, we have

$$
\left({ }_{a} I^{\alpha, \rho}{ }_{a} I^{\beta, \rho} f\right)(t)=\left({ }_{a} I^{\beta, \rho}{ }_{a} I^{\alpha, \rho} f\right)(t)=\left({ }_{a} I^{\alpha+\beta, \rho} f\right)(t) .
$$

Lemma $2([29])$ Let $0 \leq m<[\operatorname{Re}(\alpha)]+1$ and $f$ be integrable in each interval $[a, t], t>a$. Then

$$
\left({ }_{a} D^{m, \rho}{ }_{a} I^{\alpha, \rho} f\right)(t)=\left({ }_{a} I^{\alpha-m, \rho} f\right)(t) .
$$


Corollary 1 ([29]) Let $0<\operatorname{Re}(\beta)<\operatorname{Re}(\alpha)$ and $m-1<\operatorname{Re}(\beta) \leq m$. Then we have

$$
\left({ }_{a} D^{\beta, \rho}{ }_{a} I^{\alpha, \rho} f\right)(t)=\left({ }_{a} I^{\alpha-\beta, \rho} f\right)(t) .
$$

Lemma 3 ([29]) Let $f$ be integrable on $t \geq a$ and $\operatorname{Re}(\alpha)>0, \rho>0, n=[\operatorname{Re}(\alpha)]+1$. Then we have

$$
\left({ }_{a} D^{\alpha, \rho}{ }_{a}{ }^{\alpha, \rho} f\right)(t)=f(t)
$$

Lemma 4 ([29]) Let $\operatorname{Re}(\alpha)>0, n=[\operatorname{Re}(\alpha)], f \in L_{1}(a, b)$, and $\left({ }_{a} I^{\alpha, \rho} f\right)(t) \in A C^{n}[a, b]$. Then

$$
\left({ }_{a} I^{\alpha, \rho}{ }_{a} D^{\alpha, \rho} f\right)(t)=f(t)-e^{\frac{\rho-1}{\rho}(t-a)} \sum_{j=1}^{n}\left(a^{j}{ }^{j-\alpha, \rho} f\right)\left(a^{+}\right) \frac{(t-a)^{\alpha-j}}{\rho^{\alpha-j} \Gamma(\alpha+1-j)} .
$$

Definition 4 ([29]) For $0<\rho \leq 1$ and $\alpha \in \mathbb{C}$ with $\operatorname{Re}(\alpha) \geq 0$, we define the GPF derivative of Caputo type starting at $a$ by

$$
\begin{aligned}
\left({ }_{a}^{C} D^{\alpha, \rho} f\right)(t) & =\left({ }_{a} I^{n-\alpha, \rho} D^{n, \rho} f\right)(t) \\
& =\frac{1}{\rho^{n-\alpha} \Gamma(n-\alpha)} \int_{a}^{t} e^{\frac{\rho-1}{\rho}(t-\tau)}(t-\tau)^{n-\alpha-1}\left(D^{n, \rho} f\right)(\tau) d \tau,
\end{aligned}
$$

where $n=[\operatorname{Re}(\alpha)]+1$.

Proposition 2 ([29]) Let $\alpha, \beta \in \mathbb{C}$ be such that $\operatorname{Re}(\alpha)>0$ and $\operatorname{Re}(\beta)>0$. Then, for any $\rho \in(0,1]$ and $n=[\operatorname{Re}(\alpha)]+1$, we have

$$
\left({ }_{a}^{C} D^{\alpha, \rho} e^{\frac{\rho-1}{\rho} t}(t-a)^{\beta-1}\right)(x)=\frac{\rho^{\alpha} \Gamma(\beta)}{\Gamma(\beta-\alpha)} e^{\frac{\rho-1}{\rho} x}(x-a)^{\beta-1-\alpha}, \quad \operatorname{Re}(\alpha) \geq n .
$$

For $k=0,1, \ldots, n-1$, we have $\left({ }_{a}^{C} D^{\alpha, \rho} e^{\frac{\rho-1}{\rho} t}(t-a)^{k}\right)(x)=0$.

Lemma 5 ([29]) For $\rho \in(0,1]$ and $n=[\operatorname{Re}(\alpha)]+1$, we have

$$
\left({ }_{a} I^{\alpha, \rho C} D_{a}^{\alpha, \rho} f\right)(t)=f(t)-\sum_{k=0}^{n-1} \frac{\left(D^{k, \rho} f\right)(a)}{\rho^{k} \Gamma(k+1)}(t-a)^{k} e^{\frac{\rho-1}{\rho}(t-a)} .
$$

We state the following relation that links the Caputo and Riemann-Liouville GPF derivatives.

Proposition 3 ([29]) For any $\alpha \in \mathbb{C}$ with $\operatorname{Re}(\alpha)>0$ and $\rho \in(0,1], n=[\operatorname{Re}(\alpha)]+1$, we have

$$
\left({ }_{a}^{C} D^{\alpha, \rho} f\right)(t)=\left({ }_{a} D^{\alpha, \rho} f\right)(t)-\sum_{k=0}^{n-1} \frac{\rho^{\alpha-k}}{\Gamma(k+1-\alpha)}(t-a)^{k-\alpha} e^{\frac{\rho-1}{\rho}(t-a)}\left(D^{k, \rho} f\right)(a) .
$$

\section{Gronwall inequality via GPF derivative}

In this section, we prove a Gronwall inequality within GPF derivative. Besides, a particular version expressed by means of Mittag-Leffler functions is provided. 
Lemma 6 (Generalized proportional fractional Gronwall inequality) Let $\alpha, \rho>0, u(t), v(t)$ be nonnegative functions locally integrable on $[0, T)$ and $w(t)$ be a nonnegative, nondecreasing, and continuous function defined on $t \in[0, T)$ such that $w(t) \leq M$, where $M$ is a constant. If

$$
u(t) \leq v(t)+\rho^{\alpha} \Gamma(\alpha) w(t)\left({ }_{0} I^{\alpha, \rho} u\right)(t)
$$

then

$$
u(t) \leq v(t)+\int_{0}^{t}\left\{\sum_{m=1}^{\infty} \frac{(w(t) \Gamma(\alpha))^{m}}{\Gamma(m \alpha)} e^{\frac{\rho-1}{\rho}(t-s)}(t-s)^{m \alpha-1} v(s)\right\} d s, \quad t \in[0, T) .
$$

Proof Define

$$
B \phi(t)=w(t) \int_{0}^{t} e^{\frac{\rho-1}{\rho}(t-s)}(t-s)^{\alpha-1} \phi(s), \quad t \in[0, T) .
$$

It follows that $u(t) \leq v(t)+B u(t)$, which implies that $u(t) \leq \sum_{k=0}^{m-1} B^{k} v(t)+B^{m} u(t)$. We claim that

$$
B^{m} u(t) \leq \int_{0}^{t} \frac{(w(t) \Gamma(\alpha))^{m}}{\Gamma(m \alpha)} e^{\frac{\rho-1}{\rho}(t-s)}(t-s)^{m \alpha-1} u(s) d s
$$

and $B^{m} u(t) \rightarrow 0$ as $m \rightarrow \infty$ for $t \in[0, T)$. It is easy to see that (18) is valid for $m=1$. Assume that it is true for $m=k$, that is,

$$
B^{k} u(t) \leq \int_{0}^{t} \frac{(w(t) \Gamma(\alpha))^{k}}{\Gamma(k \alpha)} e^{\frac{\rho-1}{\rho}(t-s)}(t-s)^{k \alpha-1} u(s) d s .
$$

If $m=k+1$, then

$$
\begin{aligned}
B^{k+1} u(t) & =B\left(B^{k} u(t)\right) \\
& \leq w(t) \int_{0}^{t} e^{\frac{\rho-1}{\rho}(t-s)}(t-s)^{\alpha-1}\left[\int_{0}^{s} \frac{(w(s) \Gamma(\alpha))^{k}}{\Gamma(k \alpha)} e^{\frac{\rho-1}{\rho}(s-v)}(s-v)^{k \alpha-1} u(v) d v\right] d s \\
& =\frac{w^{k+1}(t) \Gamma^{k}(\alpha)}{\Gamma(k \alpha)} \int_{0}^{t}\left[\int_{v}^{t} e^{\frac{\rho-1}{\rho}(t-s)} e^{\frac{\rho-1}{\rho}(s-v)}(t-s)^{\alpha-1}(s-v)^{k \alpha-1} d s\right] u(v) d v .
\end{aligned}
$$

Upon changing variables $s=v+z(t-v)$, however, we obtain

$$
\begin{aligned}
\int_{v}^{t} e^{\frac{\rho-1}{\rho}(t-v)}(t-s)^{\alpha-1}(s-v)^{k \alpha-1} d s & =(t-v)^{k \alpha+\alpha-1} e^{\frac{\rho-1}{\rho}(t-v)} \int_{0}^{1}(1-z)^{\alpha-1} z^{k \alpha-1} d z \\
& =\frac{\Gamma(\alpha) \Gamma(k \alpha)}{\Gamma((k+1) \alpha)} e^{\frac{\rho-1}{\rho}(t-v)}(t-v)^{(k+1) \alpha-1}
\end{aligned}
$$

Therefore, (19) becomes

$$
B^{k+1} u(t) \leq \frac{w^{k+1}(t) \Gamma^{k+1}(\alpha)}{\Gamma((k+1) \alpha)} \int_{0}^{t} e^{\frac{\rho-1}{\rho}(t-v)}(t-v)^{(k+1) \alpha-1} u(v) d v .
$$


Furthermore, one can figure out that

$$
B^{m} u(t) \leq \int_{0}^{t} \frac{(M \Gamma(\alpha))^{m}}{\Gamma(m \alpha)} e^{\frac{\rho-1}{\rho}(t-s)}(t-s)^{m \alpha-1} u(s) d s \rightarrow 0 \quad \text { as } m \rightarrow \infty, t \in[0, T) .
$$

To complete the proof, we let $m \rightarrow \infty$ in

$$
u(t) \leq \sum_{k=0}^{m-1} B^{k} v(t)+B^{m} u(t) \leq v(t)+\sum_{k=1}^{m-1} B^{k} v(t)+B^{m} u(t)
$$

to reach at $u(t) \leq v(t)+\sum_{k=1}^{\infty} B^{k} v(t)$. By the help of the semigroup property and the definition of $B$, we get (17). This completes the proof.

For $w(t) \equiv b$ in Lemma 6, we have the following corollary.

Corollary 2 Let $\alpha, \rho>0, b>0, u(t), v(t)$ be nonnegative functions locally integrable on $[0, T)$ and $w(t) \equiv b \geq 0$. If

$$
u(t) \leq v(t)+\rho^{\alpha} \Gamma(\alpha) b\left({ }_{0} I^{\alpha, \rho} u\right)(t)
$$

then

$$
u(t) \leq v(t)+\int_{0}^{t}\left\{\sum_{m=1}^{\infty} \frac{(b \Gamma(\alpha))^{m}}{\Gamma(m \alpha)} e^{\frac{\rho-1}{\rho}(t-s)}(t-s)^{m \alpha-1} v(s)\right\} d s, \quad t \in[0, T) .
$$

Let $E_{\alpha}(\lambda, z)=\sum_{k=0}^{\infty} \frac{\lambda^{k} z^{k \alpha}}{\Gamma(\alpha k+1)}$ be the Mittag-Leffler function in one parameter which was introduced in [11]. The following immediate consequence of Lemma 6 plays a key role in our later analysis.

Corollary 3 Under the hypothesis of Lemma 6, assume further that $v(t)$ is a nondecreasing function for $t \in[0, T)$, then

$$
u(t) \leq v(t) E_{\alpha}(w(t) \Gamma(\alpha), t), \quad t \in[0, T) .
$$

Proof From (17) and the assumption that $v(t)$ is a nondecreasing function for $t \in[0, T)$, we may write

$$
u(t) \leq v(t)\left[1+\int_{0}^{t}\left\{\sum_{m=1}^{\infty} \frac{(w(t) \Gamma(\alpha))^{m}}{\Gamma(m \alpha)} e^{\frac{\rho-1}{\rho}(t-s)}(t-s)^{m \alpha-1}\right\} d s\right]
$$

or

$$
u(t) \leq v(t)\left[1+\sum_{m=1}^{\infty}\left(\rho^{\alpha} w(t) \Gamma(\alpha)\right)^{m} \frac{1}{\rho^{m \alpha} \Gamma(m \alpha)} \int_{0}^{t} e^{\frac{\rho-1}{\rho}(t-s)}(t-s)^{m \alpha-1} d s\right] .
$$


Using Proposition 1 and $e^{\frac{\rho-1}{\rho}(t-s)} \leq 1$, it follows that

$$
\begin{aligned}
u(t) & \leq v(t)\left[1+\sum_{m=1}^{\infty}\left(\rho^{\alpha} w(t) \Gamma(\alpha)\right)^{m} \frac{t^{m \alpha}}{\rho^{m \alpha} \Gamma(m \alpha+1)}\right] \\
& =v(t)\left[1+\sum_{m=1}^{\infty} \frac{(w(t) \Gamma(\alpha))^{m} t^{m \alpha}}{\Gamma(m \alpha+1)}\right] \\
& =v(t) \sum_{m=0}^{\infty} \frac{(w(t) \Gamma(\alpha))^{m} t^{m \alpha}}{\Gamma(m \alpha+1)} \\
& =v(t) E_{\alpha}(w(t) \Gamma(\alpha), t) .
\end{aligned}
$$

The proof is complete.

\section{Applications of Gronwall inequality via GPF derivative}

Let $\mathbb{R}^{m}$ be an $m$-dimensional Euclidean space. We prove our main results for the system

$$
\left\{\begin{array}{l}
\left({ }_{0}^{C} D^{\alpha, \rho} x\right)(t)=e^{\frac{\rho-1}{\rho} t}\left[A_{0} x(t)+A_{1} x(t-\tau)+f(t, x(t), x(t-\tau))\right], \quad t \in[0, T], \\
x(t)=\varphi(t), \quad t \in[-\tau, 0],
\end{array}\right.
$$

where ${ }_{0}^{C} D^{\alpha, \rho}$ denotes the GPF derivative of Caputo type of order $\alpha \in(0,1)$, the state vector $x:[-\tau, T] \rightarrow \mathbb{R}^{m}$, the constant matrices $A_{0}$ and $A_{1}$ are of appropriate dimensions, the nonlinearity $f:[0, T] \times \mathbb{R}^{m} \times \mathbb{R}^{m} \rightarrow \mathbb{R}^{m}$, and the initial function $\varphi:[-\tau, 0] \rightarrow \mathbb{R}^{m}$. By virtue of the results obtained in the previous sections, we prove the uniqueness and obtain an estimate for the solutions of system (24). Moreover, a numerical example is presented to demonstrate the applicability of the main results.

Let $|\cdot|$ be any Euclidean norm and $\|\cdot\|$ be the matrix norm induced by this vector. Denote by $\mathcal{C}:=C\left([-\tau, 0], \mathbb{R}^{m}\right)$ the set of all continuous functions. Clearly, the space $\mathcal{C}$ is a Banach space induced by the norm $\|z\|_{\mathcal{C}}:=\sup _{t \in[-\tau, 0]}|z(t)|$.

Throughout the remaining part of the paper, we make use of the following assumptions:

(H.1) The nonlinearity $f \in C\left([0, T] \times \mathbb{R}^{m} \times \mathbb{R}^{m}, \mathbb{R}^{m}\right)$ satisfies the Lipschitz condition.

That is, there exists a positive constant $L_{1}>0$ such that

$$
\begin{aligned}
& \|f(t, x(t), x(t-\tau))-f(t, y(t), y(t-\tau))\| \\
& \quad \leq L_{1}(\|x(t)-y(t)\|+\|x(t-\tau)-y(t-\tau)\|)
\end{aligned}
$$

for $t \in[0, T]$.

(H.2) There exists a positive constant $L_{2}$ such that $\|f(t, x(t), x(t-\tau))\| \leq L_{2}$.

In what follows, we provide a representation for the solutions of system (24) that will be useful in the subsequent analysis.

Lemma 7 The function $x:[-\tau, 0] \rightarrow \mathbb{R}^{m}$ is a solution of system (24) if and only if

$$
\left\{\begin{aligned}
x(t)= & \varphi(0) e^{\frac{\rho-1}{\rho} t} \\
& +\left({ }_{0} I^{\alpha, \rho} e^{\frac{\rho-1}{\rho} s}\left[A_{0} x(s)+A_{1} x(s-\tau)+f(s, x(s), x(s-\tau))\right]\right)(t), \quad t \in[0, T], \\
x(t)= & \varphi(t), \quad t \in[-\tau, 0] .
\end{aligned}\right.
$$


Proof For $t \in[-\tau, 0]$, it is clear that $x(t)=\varphi(t)$ is the solution of (24). We apply the operator ${ }_{0} D^{\alpha, \rho}$ on both sides of equation (25) with Proposition 1 and Lemma 3 to obtain, for $t \in$ $[0, T]$,

$$
\left({ }_{0} D^{\alpha, \rho} x\right)(t)=\varphi(0) \frac{\rho^{\alpha} e^{\frac{\rho-1}{\rho} t} t^{-\alpha}}{\Gamma(1-\alpha)}+e^{\frac{\rho-1}{\rho} t}\left[A_{0} x(t)+A_{1} x(t-\tau)+f(t, x(t), x(t-\tau))\right] .
$$

By using the relation of the Caputo and Riemann-Liouville GPF derivatives in Proposition 3, it follows that

$$
\left({ }_{0}^{C} D^{\alpha, \rho} x\right)(t)=e^{\frac{\rho-1}{\rho} t}\left[A_{0} x(t)+A_{1} x(t-\tau)+f(t, x(t), x(t-\tau))\right] .
$$

For system (24), we can see that $x(t)=\varphi(t), t \in[-\tau, 0]$. For $t \in[0, T]$, we apply the operator ${ }_{0} I^{\alpha, \rho}$ on both sides of equation (24) to get

$$
\left({ }_{0} I^{\alpha, \rho C} D_{0}^{\alpha, \rho} x\right)(t)=\left({ }_{0} I^{\alpha, \rho} e^{\frac{\rho-1}{\rho} s}\left[A_{0} x(s)+A_{1} x(s-\tau)+f(s, x(s), x(s-\tau))\right]\right)(t) .
$$

In view of Lemma 5 , one can easily see that

$$
x(t)=\varphi(0) e^{\frac{\rho-1}{\rho} t}+\left({ }_{0} I^{\alpha, \rho} e^{\frac{\rho-1}{\rho} s}\left[A_{0} x(s)+A_{1} x(s-\tau)+f(s, x(s), x(s-\tau))\right]\right)(t) .
$$

\subsection{Uniqueness of solutions}

The first main application in this paper is provided in the following theorem.

Theorem 1 Let condition (H.1) hold. If $x$ and $y$ are two solutions for system (24), then $x=y$.

Proof Let $x$ and $y$ be two solutions of system (24). Denote $z=x-y$. Then, one can easily figure out that $z(t)=0$ for $t \in[-\tau, 0]$. This implies that system (24) has a unique solution for $t \in[-\tau, 0]$.

For $t \in[0, T]$, however, we have

$$
z(t)=\left({ }_{0} I^{\alpha, \rho} e^{\frac{\rho-1}{\rho} s}\left[A_{0} z(s)+A_{1} z(s-\tau)+f(s, x(s), x(s-\tau))-f(s, y(s), y(s-\tau))\right]\right)(t) .
$$

If $t \in[0, \tau]$, then $z(t-\tau)=0$. Therefore,

$$
z(t)=\left({ }_{0} I^{\alpha, \rho} e^{\frac{\rho-1}{\rho} s}\left[A_{0} z(s)+f(s, x(s), x(s-\tau))-f(s, y(s), y(s-\tau))\right]\right)(t) .
$$

This implies

$$
\begin{aligned}
\|z(t)\| & \leq\left({ }_{0} I^{\alpha, \rho} e^{\frac{\rho-1}{\rho} s}\left[\left\|A_{0}\right\|\|z(s)\|+\|f(s, x(s), x(s-\tau))-f(s, y(s), y(s-\tau))\|\right]\right)(t) \\
& \leq\left({ }_{0} I^{\alpha, \rho} e^{\frac{\rho-1}{\rho} s}\left[\left\|A_{0}\right\|\|z(s)\|+L_{1}(\|x(s)-y(s)\|+\|x(s-\tau)-y(s-\tau)\|]\right)(t)\right. \\
& =\left({ }_{0} I^{\alpha, \rho} e^{\frac{\rho-1}{\rho} s}\left[\left(\left\|A_{0}\right\|+L_{1}\right)\|z(s)\|+L_{1}\|z(s-\tau)\|\right]\right)(t) \\
& =\left(\left\|A_{0}\right\|+L_{1}\right)\left({ }_{0} I^{\alpha, \rho} e^{\frac{\rho-1}{\rho} s}\|z(s)\|\right)(t) .
\end{aligned}
$$


By applying the result of Corollary 3, we have

$$
\|z(t)\| \leq(0) \cdot E_{\alpha}\left(\left\|A_{0}\right\|+L_{1}, t\right)
$$

which implies that $x(t)=y(t)$ for $t \in I_{\tau}$.

For $t \in[\tau, T]$, we get

$$
\begin{aligned}
z(t)= & \left({ }_{0} I^{\alpha, \rho} e^{\frac{\rho-1}{\rho} s}\left[A_{0} z(s)+f(s, x(s), x(s-\tau))-f(s, y(s), y(s-\tau))\right]\right)(t) \\
& +\left({ }_{0} I^{\alpha, \rho} e^{\frac{\rho-1}{\rho} s}\left[A_{1} z(s-\tau)\right]\right)(t) .
\end{aligned}
$$

It follows that

$$
\begin{aligned}
\|z(t)\| \leq & \left({ }_{0} I^{\alpha, \rho} e^{\frac{\rho-1}{\rho} s}\left[\left\|A_{0}\right\|\|z(s)\|+\|f(s, x(s), x(s-\tau))-f(s, y(s), y(s-\tau))\|\right]\right)(t) \\
& +\left({ }_{0} I^{\alpha, \rho} e^{\frac{\rho-1}{\rho} s}\left[\left\|A_{1}\right\|\|z(s-\tau)\|\right]\right)(t) \\
\leq & \left(\left\|A_{0}\right\|+L_{1}\right)\left({ }_{0} I^{\alpha, \rho} e^{\frac{\rho-1}{\rho} s}\|z(s)\|\right)(t)+\left(\left\|A_{1}\right\|+L_{1}\right)\left({ }_{0} I^{\alpha, \rho} e^{\frac{\rho-1}{\rho} s}\|z(s-\tau)\|\right)(t) .
\end{aligned}
$$

Let $\bar{z}(t)=\sup _{\theta \in[-\tau, 0]}\|z(t+\theta)\|$, then we get

$$
\begin{aligned}
\bar{z}(t) & \leq\left(\left\|A_{0}\right\|+L_{1}\right)\left({ }_{0} I^{\alpha, \rho} e^{\frac{\rho-1}{\rho} s} \bar{z}(s)\right)(t)+\left(\left\|A_{1}\right\|+L_{1}\right)\left({ }_{0} I^{\alpha, \rho} e^{\frac{\rho-1}{\rho} s} \bar{z}(s)\right)(t) \\
& \leq\left(\left\|A_{0}\right\|+\left\|A_{1}\right\|+2 L_{1}\right)\left({ }_{0} I^{\alpha, \rho} e^{\frac{\rho-1}{\rho} s} \bar{z}(s)\right)(t) .
\end{aligned}
$$

By applying the result of Corollary 3, we obtain

$$
\|z(t)\| \leq \bar{z}(t) \leq(0) \cdot E_{\alpha}\left(\left\|A_{0}\right\|+\left\|A_{1}\right\|+2 L_{1}, t\right) .
$$

Hence, we end up with $x(t)=y(t)$ for $t \in[-\tau, T]$.

\subsection{Bound for solutions}

In this subsection, we provide a bound for the solution of system (24).

Theorem 2 Let condition (H.2) hold. Then the following estimate for the solution $x(t)$ of system (24) is valid:

$$
\|x(t)\| \leq\left[\|\varphi\|+\left(L_{2}+\left(\left\|A_{0}\right\|+\left\|A_{1}\right\|\right)\|\varphi\|\right) \frac{t^{\alpha}}{\rho^{\alpha} \Gamma(\alpha+1)}\right] E_{\alpha}\left(\left(\left\|A_{0}\right\|+\left\|A_{1}\right\|\right) \Gamma(\alpha), t\right) .
$$

Proof For $t \in[0, T]$, the solution of system (24) has the form

$$
x(t)=\varphi(0) e^{\frac{\rho-1}{\rho} t}+\left({ }_{0} I^{\alpha, \rho} e^{\frac{\rho-1}{\rho} s}\left[A_{0} x(s)+A_{1} x(s-\tau)+f(s, x(s), x(s-\tau))\right]\right)(t) .
$$

Using the fact $e^{\frac{\rho-1}{\rho} t} \leq 1$ for all $t \in[0, T]$, it follows that

$$
\begin{aligned}
\|x(t)\| \leq & \|\varphi(0)\|+\left({ }_{0} I^{\alpha, \rho} e^{\frac{\rho-1}{\rho} s}\left\|A_{0} x(s)+A_{1} x(s-\tau)+f(s, x(s), x(s-\tau))\right\|\right)(t) \\
\leq & \|\varphi\|+\left\|A_{0}\right\|\left({ }_{0} I^{\alpha, \rho} e^{\frac{\rho-1}{\rho} s}\|x(s)\|\right)(t)+\left\|A_{1}\right\|\left({ }_{0} I^{\alpha, \rho} e^{\frac{\rho-1}{\rho} s}\|x(s-\tau)\|\right)(t) \\
& +\left({ }_{0} I^{\alpha, \rho} e^{\frac{\rho-1}{\rho} s}\|f(s, x(s), x(s-\tau))\|\right)(t) .
\end{aligned}
$$


By assumption (H.2) and Proposition 1, the above inequality can be rewritten as follows:

$$
\begin{aligned}
\|x(t)\| \leq & \|\varphi\|+\left(\left\|A_{0}\right\|+\left\|A_{1}\right\|\right)\left({ }_{0} I^{\alpha, \rho} e^{\frac{\rho-1}{\rho} s}\left[\sup _{\theta \in[-\tau, 0]}\|x(s+\theta)\|+\|\varphi\|\right]\right)(t) \\
& +L_{2}\left({ }_{0} I^{\alpha, \rho} e^{\frac{\rho-1}{\rho} s}(1)\right)(t) \\
= & \|\varphi\|+\left(L_{2}+\left(\left\|A_{0}\right\|+\left\|A_{1}\right\|\right)\|\varphi\|\right) \frac{t^{\alpha}}{\rho^{\alpha} \Gamma(\alpha+1)} \\
& +\left(\left\|A_{0}\right\|+\left\|A_{1}\right\|\right)\left({ }_{0} I^{\alpha, \rho} e^{\frac{\rho-1}{\rho} s}\left[\sup _{\theta \in[-\tau, 0]}\|x(s+\theta)\|\right]\right)(t) .
\end{aligned}
$$

Let $v(t)=\|\varphi\|+\left(L_{2}+\left(\left\|A_{0}\right\|+\left\|A_{1}\right\|\right)\|\varphi\|\right) \frac{T^{\alpha}}{\rho^{\alpha} \Gamma(\alpha+1)}$, then $v$ is a nondecreasing function. Therefore, Corollary 3 with $w(t)=\left\|A_{0}\right\|+\left\|A_{1}\right\|$ implies that

$$
\|x(t)\| \leq \sup _{\theta \in[-\tau, 0]}\|x(t+\theta)\| \leq v(t) E_{\alpha}\left(\left(\left\|A_{0}\right\|+\left\|A_{1}\right\|\right) \Gamma(\alpha), t\right) .
$$

Hence, the solution $x$ of (24) satisfies the estimate

$$
\|x(t)\| \leq\left[\|\varphi\|+\left(L_{2}+\left(\left\|A_{0}\right\|+\left\|A_{1}\right\|\right)\|\varphi\|\right) \frac{t^{\alpha}}{\rho^{\alpha} \Gamma(\alpha+1)}\right] E_{\alpha}\left(\left(\left\|A_{0}\right\|+\left\|A_{1}\right\|\right) \Gamma(\alpha), t\right) .
$$

The proof is complete.

Example 1 Consider the nonlinear delay proportional fractional system of the form

$$
\left\{\begin{array}{l}
\left({ }_{0}^{C} D^{\frac{1}{2}, \frac{1}{3}} x\right)(t)=e^{-2 t}[3 x(t)+x(t-2)+2 \cos x(t)-\cos x(t-2)], \quad t \in[0,1], \\
x(t)=\sin 2 t, \quad t \in[-2,0] .
\end{array}\right.
$$

This corresponds to equation (24) with $\alpha=1 / 2, \rho=1 / 3, A_{0}=3, A_{1}=1, T=1$, and $\tau=2$. The nonlinearity has the form $f(t, x(t), x(t-\tau))=2 \cos x(t)-\cos x(t-2)$. Therefore, we have

$$
\begin{aligned}
& \|f(t, x(t), x(t-\tau))-f(t, y(t), y(t-\tau))\| \\
& \quad=\|2 \cos x(t)-\cos x(t-2)-2 \cos y(t)+\cos y(t-2)\| \\
& \quad \leq 2(\|\cos x(t)-\cos y(t)\|+\|\cos x(t-2)-\cos y(t-2)\|) .
\end{aligned}
$$

Then assumption (H.1) holds with $L_{1}=2$. By the consequence of Lemma 7, system (36) has a unique solution. Moreover,

$$
\|f(t, x(t), x(t-\tau))\|=\|2 \cos x(t)-\cos x(t-2)\| \leq 3,
$$

which implies that assumption (H.2) is satisfied with $L_{2}=3$. By Theorem 2, the solution $x$ of system (36) has the estimate

$$
\|x(t)\| \leq\left[1+\frac{24 \sqrt{3}}{\sqrt{\pi}} t^{\frac{1}{2}}\right] \sum_{k=0}^{\infty} \frac{(4 \sqrt{\pi})^{k} t^{\frac{k}{2}}}{\Gamma\left(\frac{k}{2}+1\right)} .
$$




\section{Conclusion}

One of the most important issues in the theory of differential equations is finding qualitative properties of these equations. Integral equations are significant tools that facilitate exploring such properties. In this paper, we established the Gronwall inequality within the scope of fractional proportional operators. This inequality helped in proving the uniqueness of solutions to delay differential equations involving fractional proportional derivatives and in finding bounds of these solutions. In addition to what has been done in this paper, one can use the established Gronwall inequality to discuss some other qualitative properties of these solutions such as the stability in the sense of Ulam or Ulam-Hyers.

\section{Acknowledgements}

The authors would like to express their sincere thanks to the editor and the anonymous referees for their recommendations. We believe that their valuable comments have improved the contents of the paper.

\section{Funding}

The first and second authors would like to thank Prince Sultan University for funding this work through research group Nonlinear Analysis Methods in Applied Mathematics (NAMAM), group number RG-DES-2017-01-17.

Availability of data and materials

Not applicable.

Competing interests

The authors declare that they have no competing interests.

Authors' contributions

All authors contributed equally and significantly to this paper. All authors have read and approved the final version of the manuscript.

\section{Author details}

${ }^{1}$ Department of Mathematics and General Sciences, Prince Sultan University, Riyadh, Saudi Arabia. ${ }^{2}$ Department of Mathematics, Çankaya University, Ankara, Turkey. ${ }^{3}$ Department of General Education, Navamindradhiraj University, Bangkok, Thailand.

\section{Publisher's Note}

Springer Nature remains neutral with regard to jurisdictional claims in published maps and institutional affiliations.

Received: 12 February 2019 Accepted: 4 April 2019 Published online: 11 April 2019

\section{References}

1. Hardy, G.H., Littlewood, J.E., Pólya, G.: Inequalities. Cambridge University Press, Cambridge (1952)

2. Bainov, D.D., Simeonov, P.: Integral Inequalities and Applications. Mathematics and Its Applications. Springer, Berlin (1992)

3. Cloud, J.M., Drachman, C.B., Lebedev, P.L.: Inequalities with Applications to Engineering. Springer, Berlin (2014)

4. Gronwall, T.H.: Note on the derivatives with respect to a parameter of the solutions of a system of differential equations. Ann. Math. 20, 292-296 (1919)

5. Rasmussen, D.L.: Gronwall's inequality for functions of two independent variables. J. Math. Anal. Appl. 55(2), 407-417 (1976)

6. Dragomir, S.S.: Some Gronwall Type Inequalities and Applications. RGMIA Monographs, Victoria University (2003)

7. Lin, X.: A note on Gronwall's inequality on time scales. Abstr. Appl. Anal. 2014, Article ID 623726 (2014)

8. Wang, W., Feng, Y., Wang, Y.: Nonlinear Gronwall-Bellman type inequalities and their applications. Mathematics 5, 31 (2017). https://doi.org/10.3390/math5020031

9. Hilfer, R.: Applications of Fractional Calculus in Physics. Word Scientific, Singapore (2000)

10. Debnath, L.: Recent applications of fractional calculus to science and engineering. Int. J. Math. Math. Sci. 2003(54), 3413-3442 (2003)

11. Kilbas, A., Srivastava, H.M., Trujillo, J.J.: Theory and Application of Fractional Differential Equations. North Holland Mathematics Studies, vol. 204 (2006)

12. Magin, R.L.: Fractional Calculus in Bioengineering. Begell House Publishers, Danbury (2006)

13. Samko, S.G., Kilbas, A.A., Marichev, O.I.: Fractional Integrals and Derivatives: Theory and Applications. Gordon \& Breach, Yverdon (1993)

14. Podlubny, I.: Fractional Differential Equations. Mathematics in Science and Engineering, vol. 198. Academic Press, San Diego (1999)

15. Caputo, M., Fabrizio, M.: A new definition of fractional derivative without singular kernel. Prog. Fract. Differ. Appl. 1, 73-85 (2015)

16. Atangana, A., Baleanu, D.: New fractional derivatives with non-local and non-singular kernels. Therm. Sci. 20, 757-763 (2016) 
17. Ye, H., Gao, J., Ding, Y.: A generalized Gronwall inequality and its application to a fractional differential equation. J. Math. Anal. Appl. 328, 1075-1081 (2007)

18. Ferreira, R.A.C.: A discrete fractional Gronwall inequality. Proc. Am. Math. Soc. 5, 1605-1612 (2012)

19. Abdeljawad, T., Alzabut, J., Baleanu, D.: A generalized q-fractional Gronwall inequality and its applications to nonlinear delay q-fractional difference systems. J. Inequal. Appl. 2016, 240 (2016)

20. Zhang, Z., Wei, Z:: A generalized Gronwall inequality and its application to fractional neutral evolution inclusions. J. Inequal. Appl. 2016, 45 (2016)

21. Sarikaya, M.Z:: Gronwall type inequalities for conformable fractional integrals. Konuralp J. Math. 4(2), 217-222 (2016)

22. Alzabut, J., Abdeljawad, T.: A generalized discrete fractional Gronwall inequality and its application on the uniqueness of solutions for nonlinear delay fractional difference system. Appl. Anal. Discrete Math. 12, 36-48 (2018)

23. Abdeljawad, T:: A Lyapunov type inequality for fractional operators with nonsingular Mittag-Leffler kernel. J. Inequal. Appl. 2018, 143 (2018)

24. Abdeljawad, T.: Fractional operators with exponential kernels and a Lyapunov type inequality. Adv. Differ. Equ. 2017 $313(2017)$

25. Abdeljawad, T., Alzabut, J., Jarad, F.: A generalized Lyapunov-type inequality in the frame of conformable derivatives. Adv. Differ. Equ. 2017, 321 (2017)

26. Abdeljawad, T., Agarwal, R., Alzabut, J., Jarad, F., Özbekler, A.: Lyapunov-type inequalities for mixed non-linear forced differential equations within conformable derivatives. J. Inequal. Appl. 2018, 143 (2018)

27. Abdeljawad, T., Madjidi, F:: Lyapunov-type inequalities for fractional difference operators with discrete Mittag-Leffler kernel of order $2<\alpha<5 / 2$. Eur. Phys. J. Spec. Top. 226(16-18), 3355-3368 (2017)

28. Jarad, F., Abdeljawad, T., Hammouch, Z.: On a class of ordinary differential equations in the frame of Atangana-Baleanu fractional derivative. Chaos Solitons Fractals 117, 16-20 (2018)

29. Jarad, F., Abdeljawad, T., Alzabut, J.: Generalized fractional derivatives generated by a class of local proportional derivatives. Eur. Phys. J. Spec. Top. 226(16-18), 3457-3471 (2017)

30. Abdeljawad, T:: On conformable fractional calculus. J. Comput. Appl. Math. 279, 57-66 (2015)

31. Khalil, R., Al Horani, M., Yousef, A., Sababheh, M.: A new definition of fractional derivative. J. Comput. Appl. Math. 264, 65-70 (2014)

32. Anderson, D.R., Ulness, D.J.: Newly defined conformable derivatives. Adv. Dyn. Syst. Appl. 10(2), 109-137 (2015)

33. Anderson, D.R.: Second-order self-adjoint differential equations using a proportional-derivative controller. Commun. Appl. Nonlinear Anal. 24, 17-48 (2017)

\section{Submit your manuscript to a SpringerOpen ${ }^{\circ}$ journal and benefit from:}

- Convenient online submission

- Rigorous peer review

- Open access: articles freely available online

- High visibility within the field

- Retaining the copyright to your article

Submit your next manuscript at $\gg$ springeropen.com 